\title{
Estabilidad de la hidroxocobalamina en agua para inyección como antídoto contra cianuros. Elaborado de Farmacia Militar
}

\author{
Sánchez Ramos JJ. ${ }^{1}$
}

Sanid. mil. 2012; 68 (2): 87-96; ISSN: 1887-8571

RESUMEN. Introducción: Los cianuros han sido utilizados como agentes de guerra química, y hoy se consideran una amenaza terrorista real. Son también la principal causa de muerte en la intoxicación por inhalación de humo de incendios en espacios cerrados. El éxito en el tratamiento de la intoxicación depende de la rapidez con la que se administren los antídotos, y con la que estos fijen el ión cianuro. La hidroxocobalamina parece ser un antídoto más apropiado que el nitrito sódico para el tratamiento empírico de la intoxicación por inhalación de humo y otras supuestas intoxicaciones por cianuros en el ámbito extra-hospitalario. Se ha estudiado la estabilidad de la hidroxocobalamina en agua para inyección a la dosis como antídoto contra cianuros y en cuatro tipos de envases diferentes. Material y Métodos: La solución inyectable se preparó por vía aséptica sin esterilización terminal, y según las especificaciones de la monografía oficial de la farmacopea de los Estados Unidos. Los cuatro tipos de envase primario utilizados fueron: ampollas de vidrio, bolsas y viales de polipropileno semirígido, y viales de plástico rígido fabricados en un copolímero olefínico complejo (COC). La determinación del contenido en hidroxocobalamina de las soluciones bajo estudio en función del tiempo, conservadas a $4^{\circ} \mathrm{C}, 25^{\circ} \mathrm{C}$ y $37^{\circ} \mathrm{C}$, se 1 levó a cabo mediante cromatografía líquido-líquido de alta resolución y espectroscopia de absorción molecular. Se han utilizado otras técnicas analíticas complementarias para caracterizar la solución. Resultados: A $4^{\circ} \mathrm{C}$ el contenido en hidroxocobalamina de las muestras estudiadas permaneció invariable (media 99,81\% $\pm 0,69 \% ; \mathrm{p}=0,95 ; \mathrm{n}=7 ; \mathrm{t}=472$ días). Resultados muy similares se obtuvieron para las muestras a $25^{\circ} \mathrm{C}$ (media 100,85\% $\pm 1,79 \% ; \mathrm{p}=0,95 ; \mathrm{n}=6 ; \mathrm{t}=353$ días). A $37^{\circ} \mathrm{C}$ se produce una ligera disminución en el contenido en hidroxocobalamina de la solución envasada en ampollas (media $=92,87 \% \pm 6,08 \% ; \mathrm{p}=0,95 ; \mathrm{n}=4 ; \mathrm{t}=472$ días). La solución envasada en bolsas de polipropileno sufrió un efecto de concentración por pérdida de agua a través del envase. En los dos tipos de viales estudiados el contenido en hidroxocobalamina de la solución se mantuvo en el rango de aceptación especificado en la farmacopea: 102,09\% \pm 0,47\% a $4^{\circ} \mathrm{C}$ y $102,35 \% \pm 0,60 \%$ a $37^{\circ} \mathrm{C}$ para los viales COC; $101,64 \% \pm 2,16 \%$ a $4^{\circ} \mathrm{C}$ y $101,19 \pm 1,75 \%$ a $37^{\circ} \mathrm{C}$, para los viales de polipropileno $(\mathrm{p}=0,95)$. El autoclavado de la solución a $115^{\circ} \mathrm{C}$ durante 30 minutos redujo el porcentaje de hidroxocobalamina a valores fuera del rango de conformidad (95-115\%). Conclusiones: La solución inyectable de cloruro de hidroxocobalamina en agua para inyección a la concentración indicada como antídoto N.B.Q. contra cianuros, lista para administrar, es estable a temperaturas inferiores a $25^{\circ} \mathrm{C}$, al menos durante un periodo de 15 meses. Por su inercia química y propiedades físicas, los viales de polipropileno constituyen el envase más adecuado para esta solución, que no puede ser autoclavada, y debe ser preparada por vía aséptica sin esterilización terminal.

PALABRAS CLAVE: Hidroxocobalamina inyectable, Estabilidad hidroxocobalamina, Antídotos cianuros, NBQ, Terrorismo, Guerra química, Inhalación de humo.

\section{Stability of hydroxocobalamin in water for injection as cyanide antidote}

SUMMARY. Background: Cyanide has been used as an agent for chemical warfare, and today it is also a credible terrorism threat agent. Cyanide poisoning is the first cause of death in victims of smoke inhalation from enclosed space fires. Successful treatment for acute cyanide poisoning depends upon rapid antidotes administration, and fixation of the cyanide ion by antidotes. Hydroxocobalamin seems to be a more appropriate antidote than sodium nitrite for empiric treatment of smoke inhalation and other suspected cyanide intoxication victims in the out-of-hospital setting. Stability of hydroxocobalamin in water for injection at cyanide antidote dose has been studied throughout the time and in four types of containers. Methods: The parenteral solution was prepared by aseptic procedures without terminal sterilization, and according to the United States Pharmacopoeia (USP) specifications. Four types of primary containers were used: glass ampoules, semi-rigid polypropylene bags and vials, and rigid plastic vials made of COC. Hydroxocobalamin content and other parameters from the studied solutions, conserved at $4^{\circ} \mathrm{C}, 25^{\circ} \mathrm{C}$ and $37^{\circ} \mathrm{C}$ was carried out using molecular spectrometry, liquid-liquid chromatography and others assays. Results: A $4^{\circ} \mathrm{C}$ the hydroxocobalamin content in the studied samples was invariable (mean 99,81\% $\pm 0,69 \% ; \mathrm{p}=0,95 ; \mathrm{n}=7 ; \mathrm{t}=472$ days). Very similar results were obtained for samples at $25^{\circ} \mathrm{C}$ (mean $100,85 \% \pm 1,79 \% ; \mathrm{p}=0,95 ; \mathrm{n}=6 ; \mathrm{t}=353$ days). At $37^{\circ} \mathrm{C}$ there was an small decrease in the ampoules hydroxocobalamin content (mean $=92,87 \% \pm 6,08 \% ; p=0,95 ; n=4 ; t=472$ days). The solution in polypropylene bags suffered a concentration due to water permeation through the container. Both types of vials had hydroxocobalamin contents in the USP specifications (95-115\%). Steam sterilization at $115^{\circ} \mathrm{C}$ for 30 minutes turned hydroxocobalamin content out of specifications. Conclusion: The parenteral solution of hydroxocobalamin in water for injection at the dose of $\mathrm{NBC}$ cyanide antidote, ready to use, is stable at temperature below $25^{\circ} \mathrm{C}$, at least for 15 months. Because of the chemical inertia and physical properties, the polypropylene vials are the more appropriated

${ }^{1}$ Tcol. Farmacéutico. Centro Militar de Farmacia de la Defensa. Córdoba.

Dirección para correspondencia: Centro Militar de Farmacia de la Defensa (Córdoba). Avenida de Rabanales, 1. 14010-Córdoba.jsanram@oc.mde.es

Recibido: 28 de junio de 2011

Aceptado: 20 de marzo de 2012 containers for this parenteral solution, which has to be prepared by aseptic procedures without terminal sterilization.

KEY WORDS: Hydroxocobalamin injection, Hydroxocobalamin stability, Cyanide antidotes, NBQ, Terrorism, Chemical warfare, Smoke inhalation. 


\section{INTRODUCCIÓN}

Los cianuros y sus productos químicos derivados son ampliamente utilizados en la industria como intermediarios de síntesis en multitud de procesos químicos e industriales en los que se obtienen materiales plásticos o sintéticos. Estos materiales son después empleados en la construcción, industria automovilística, fabricación de mobiliario, decoración, etc. La utilización industrial masiva ha sido causa de numerosos accidentes durante su transporte y manipulación industrial y ocupacional $1^{1,2}$.

El ácido cianhídrico y sus derivados, junto al monóxido de carbono, son la principal causa de intoxicación por la inhalación de humo en los incendios en espacios cerrados. Se estima que son responsables de más del 80 por ciento de las muertes causadas por incendios domésticos. En ellos, el cianuro y sus derivados se producen por la combustión incompleta precisamente de aquellos materiales plásticos o sintéticos en los que interviene durante su producción ${ }^{1-4}$.

Desde el punto de vista militar, el ácido cianhídrico y los compuestos cianógenos se consideran agentes de guerra química o agresivos N.B.Q. (Nucleares, Biológicos y Químicos). Están encuadrados en los manuales de Defensa N.B.Q. en un solo grupo con la denominación "Agentes Cianógenos" o "Tóxicos Sanguíneos", este último término en desuso 5 .

La amenaza del terrorismo a gran escala ha hecho que los agentes cianógenos se consideren también una amenaza para la población civil. Su fácil y amplia disponibilidad les confiere potencialidad como arma terrorista real, ya utilizada en este contexto ${ }^{6-9}$.

La potencialidad de estos agentes como arma química ha llevado a la mayoría de los países de nuestro entorno a incorporar antídotos contra cianuros en las reservas o stock farmacéuticos de interés estratégico nacional.

Aunque la intoxicación por cianuros puede ser también voluntaria (suicidio o asesinato), las circunstancias más frecuentes son la inhalación de humo de incendios y los accidentes industriales y ocupacionales.

Son altamente tóxicos por inhalación, ingestión o por contacto. Ejercen su acción interfiriendo en el transporte de oxígeno a nivel molecular y celular al combinarse con el ión férrico de la enzima ferricitocromoxidasa de la mitocondria. Ello conduce a un metabolismo celular anaeróbico y acidosis metabólica por aumento del lactato en sangre, principal indicador sanguíneo de la intoxicación por cianuros. El cerebro y el corazón son los órganos más ricos en esa enzima. Los primeros síntomas son neurológicos y cardiovasculares. La muerte ocurre en 6 u 8 minutos después de la inhalación de una dosis letal. El diagnóstico rápido y el tratamiento con antídotos puede salvar al intoxicado ${ }^{5,10}$.

No obstante, el polimorfismo clínico de la intoxicación aguda y su baja frecuencia hacen que pueda pasar desapercibida para el personal de urgencias médicas. Tampoco existe ampliamente disponible un ensayo sanguíneo rápido y confirmativo para el diagnóstico de la intoxicación. El diagnóstico puede ser difícil, y la decisión de administrar un antídoto también implica riesgos, por lo que el antídoto de elección debería ser aquel que careciera de efectos secundarios, incluso en ausencia de intoxicación ${ }^{11}$.

El mecanismo de acción de los antídotos contra cianuros se basa en que la unión cianuro-enzima es reversible. Su reactiva- ción se consigue por depleción del cianuro intracelular hacia el espacio extracelular, al retirar el antídoto el cianuro circulante. Esto puede ocurrir por tres mecanismos de acción diferentes cuyos representantes más característicos son el tiosulfato sódico, el nitrito sódico y la hidroxocobalamina ${ }^{5}$.

En el primer mecanismo, el tiosulfato sódico reacciona con el ión cianuro circulante en sangre para formar tiocianato estable y no tóxico que se elimina por la orina. El tiosulfato sódico debe administrase siempre como complemento y en combinación con cualquier otro antídoto para la intoxicación por cianuros. Es seguro y eficaz, aunque algo lento en su acción ${ }^{1}$.

En el segundo mecanismo, los agentes metahemoglobinizantes como el nitrito sódico oxidan el hierro ferroso de la hemoglobina sanguínea a hierro férrico. Se forma metahemoglobina con gran apetencia por el cianuro para formar cianometahemoglobina no tóxica. Son antídotos potentes, pero influyen en la disponibilidad de oxígeno en los tejidos al transformar la hemoglobina disponible en metahemoglobina. Se deben administrar siempre en conjunción con el tiosulfato sódico.

El nitrito sódico y el tiosulfato sódico son antídotos contra cianuros de uso más extendido y tradicional, aunque con mayor incidencia de efectos secundarios. El Petitorio de Farmacia del Ministerio de Defensa ${ }^{12}$, contempla desde hace más de 15 años la fabricación de los Elaborados Tiosulfato sódico DEF Inyectable, 12,5 gramos en $50 \mathrm{ml}$, envase de 1 vial y Nitrito sódico DEF Inyectable, $300 \mathrm{mg}$ en $10 \mathrm{ml}$, envase de 10 ampollas. Ambos forman parte de la dotación de medicamentos de las Fuerzas Armadas Españolas en Misiones y Operaciones de Mantenimiento de la Paz, para la protección del contingente nacional y multinacional, y para protección civil. También forman parte de las denominadas Reservas de Guerra, un stock farmacéutico militar con medicamentos de interés estratégico y en cantidad suficiente para atender una emergencia nacional. El nitrito sódico y el tiosulfato sódico han sido tradicionalmente contemplados por los ejércitos de nuestro entorno como los antídotos NBQ de elección contra cianuros como agentes de guerra química ${ }^{5,13}$.

La hidroxocobalamina representa el tercer mecanismo de acción de los antídotos contra cianuros. Se combina con el cianuro circulante para formar cianocobalamina (vitamina $\mathrm{B}_{12}$ ), un compuesto de coordinación o quelato estable y no tóxico que se excreta por la orina. Debe administrarse por vía intravenosa a dosis muy altas si se comparan con las habituales cuando la acción es como vitamina B12 ( 5 gramos frente a 1 miligramo). Esta diferente magnitud de dosis dependiente de la indicación terapéutica, 5.000 veces superior como antídoto contra cianuros, debe ser considerada en la selección de las presentaciones comerciales de hidroxocobalamina. Frente al nitrito sódico, la hidroxocobalamina está especialmente indicada como antídoto contra cianuros cuando la intoxicación se produce por la inhalación de humo de incendios en locales cerrados (combustión de plásticos) y es concomitante con la de monóxido de carbono. En estos casos, la administración de agentes metahemoglobinizantes como el nitrito sódico puede agravar la intoxicación y están contraindicados por reducir la capacidad de transporte de oxígeno de la víctima. La hidroxocobalamina está también indicada cuando la intoxicación por cianuros es supuesta y no está confirmada, pues en general es segura y bien tolerada. Es aquí donde aventaja a 


\section{Estabilidad de la hidroxocobalamina en agua para inyección como antídoto contra cianuros...}

otros antídotos, pues en su mecanismo de acción no compromete el transporte de oxígeno a nivel molecular ${ }^{13}$.

La hidroxocobalamina es el principio activo del preparado Cyanokit $^{\mathrm{R}}$, en el que se presenta en forma de polvo para reconstituir en el momento de la administración (preparación extemporánea $)^{14}$.

Por definición, según la United States Pharmacopoeia (U.S.P. $)^{15}$, la inyección de hidroxocobalamina es una solución estéril de hidroxocobalamina en agua para inyección que contendrá no menos del $95 \%$ y no más del $115 \%$ de la cantidad etiquetada.

En este trabajo se ha estudiado la estabilidad de la solución estéril de cloruro de hidroxocobalamina en agua para inyección, sin excipientes adicionales, a la dosis habitual como antitodo contra cianuros (2,5 g en $100 \mathrm{ml})$. Se ha estudiado el comportamiento de la solución en varios tipos de envases farmacéuticos y se ha evaluado el contenido a diferentes temperaturas en función del tiempo. Se pretende disponer de una solución inyectable de hidroxocobalamina como antídoto contra cianuros, lista para administrar por vía intravenosa, sin necesidad de reconstitución o trasvase de contenidos, y en el envase más adecuado para su uso en el ámbito de las Fuerzas Armadas.

\section{MATERIAL Y MÉTODOS}

\section{Materia prima}

Se ha utilizado cloruro de hidroxocobalamina suministrado por la firma Impex Química S. A. en el Mayo de 2008. Fabricante: Hebei Huarong Pharmaceutical Co., Ltd. . Cumple las especificaciones de la Farmacopea Europea 7.0 Edición (2010) páginas 2204-2205.

\section{Método de preparación de la solución inyectable de hidroxocobalamina}

Según la monografía oficial de la farmacopea de los Estados Unidos ${ }^{15}$, la solución inyectable es una solución de hidroxocobalamina en agua para inyección. Por ello, y teniendo en cuenta la dosis como antídoto (2,5 gramos en 100 mililitros) las soluciones preparadas tuvieron siempre la misma concentración: 25 miligramos por mililitro. La pesada se realizó directamente sobre un vaso de precipitados limpio, seco y previamente tarado en balanza de precisión marca $S$ caltec $^{R}$ modelo SBA 32, con apreciación de décimas de miligramo.

La disolución se efectuó utilizando agua ultrapura (conductividad 0,055 micro siemens, estéril y apirógena; TOC $<30 \mathrm{ppb}$ ) producida por un equipo Milli- $Q$ Integral $3^{R}$ de la firma Millipore $^{\mathrm{R}}$. Además de la monitorización en línea del carbono orgánico total (COT) en dicho equipo, este parámetro se controló también mediante un equipo Sievers modelo 900 Portable TOC Analyzer de General Electric.

El cloruro de hidroxocobalamina es fácilmente soluble en agua. Sobre el vaso de precipitados con el peso exacto del principio activo se añadió el 50 por ciento del agua del lote, y se efectuó la disolución con la ayuda de una varilla de vidrio. La solución obtenida se vertió sobre un matraz aforado limpio y seco, de volumen apropiado. El vaso de precipitados fue lavado con pequeñas fracciones de agua para inyectables que fueron añadidas al matraz. Finalmente se efectuó el enrase a volumen.

\section{Ajuste del pH a la solución preparada}

Para cada determinación, el $\mathrm{pH}$-metro fue calibrado con patrones certificados de la firma $\operatorname{Crison}^{R}(\mathrm{pH} 4.00, \mathrm{pH} 7.00$ y $\mathrm{pH}$ 9.00). La solución preparada contenida en el matraz fue vertida sobre un vaso de precipitados de capacidad adecuada, limpio y seco. El pH obtenido por disolución directa estuvo siempre entre 2,60 y 2,70. Según las especificaciones de la United States Pharmacopoeia (U.S.P. $)^{15}$ para Hidroxocobalamin Injection, el $\mathrm{pH}$ de la solución inyectable debe estar comprendido entre 3,5 y 5,0. Por ello fue necesario realizar el ajuste de $\mathrm{pH}$ de las soluciones preparadas. Se utilizó una micropipeta para la adición de microgotas de una solución de hidróxido sódico $1 \mathrm{~N}$ hasta obtener un $\mathrm{pH}$ final de 4.00. La solución estuvo entonces preparada y lista para ser dosificada.

\section{Tipos de envase y materiales a ensayar}

- Ampollas de vidrio ( sin color) de primera clase hidrolítica, fabricadas por la firma Agrado S.L. . El cierre de cada ampolla ensayada se realizó manualmente al fuego de la llama de mechero. Por sus características de estanqueidad, inercia química y propiedades barrera, las soluciones envasadas en ampollas y conservadas convenientemente, mostraron estabilidad óptima y fueron tomadas como referencia analítica.

- Bolsas de suero en polipropileno (PP) fabricadas por la firma Promens Medical Packaging A/S y suministradas por Suphatec, S.L. Este fabricante y proveedor suministran también los viales de material plástico en calidad farmacéutica que se verán en el siguiente apartado. Las bolsas de suero en polipropileno (material plástico) son flexibles y casi transparente. Se presentan limpias, estériles, y apirógenas, cumpliendo las especificaciones de la Farmacopea Española ${ }^{17}$ para envases parenterales.

- Viales fabricados en un Copolímero Olefínico Complejo (COC). Son viales de material plástico, rígidos, totalmente transparentes, en colores "blanco" o "ámbar", según el caso. Resisten la esterilización con vapor de agua a $121^{\circ} \mathrm{C}$ durante 20 minutos (autoclavado) y se presentan limpios, estériles y apirógenos de conformidad con las especificaciones de la Farmacopea Española ${ }^{17}$.

- Viales de polipropileno: Son también viales de material plástico, semirrígidos, casi transparentes, en colores blanco o ámbar. Se presentan limpios, estériles y apirógenos fabricados conforme a la Farmacopea Española.

- Tapón de caucho y cápsula de aluminio tipo flip-off fabricados por West Pharmaceutical: a) Tapón de caucho de $20 \mathrm{~mm}$, artículo 1298. Código WPS 7001-7831. Cumple las especificaciones de la Farmacopea Española para los cierres para envases farmacéuticos; b) Cápsula de aluminio tipo flip-off, de $20 \mathrm{~mm}$, artículo 5199 / 3768 color gris. Código WPS: 5920-6312. Con excepción de las ampollas de vidrio, los demás envases estudiados 


\section{J. J. Sánchez Ramos}

fueron cerrados con el tapón de caucho y la cápsula de aluminio tipo flip-off referenciados.

\section{Dosificación de envases}

Tras el ajuste de $\mathrm{pH}$ de la solución preparada, esta fue esterilizada mediante filtración por membranas de 0,45 micras (Millipore, referencia B1714Y). Desde ese momento se trabajó en condiciones asépticas y bajo campana de flujo laminar.

Las muestras para los ensayos de estabilidad en viales y bolsas se obtuvieron dosificando para cada lote de ensayo, el número de envases necesario para los ensayos programados en el tiempo. Para cada tipo de envase (bolsa de polipropileno, vial COC o vial de polipropileno) se preparó un solo lote. Por el alto coste de la hidroxocobalamina los envases fueron dosificados con $10 \mathrm{ml}$ de solución, aun cuando su capacidad total era de 100 mililitros. Fueron cerrados con tapón de caucho limpio y estéril, y cápsula de aluminio tipo flip-off. En las diferentes condiciones de almacenamiento $\left(4^{\circ} \mathrm{C}, 25^{\circ} \mathrm{C}\right.$ y $\left.37^{\circ} \mathrm{C}\right)$ cada envase se mantuvo boca abajo para que la solución estuviese siempre en contacto con el tapón.

De cada lote se reservó un cierto volumen de solución preparada para dosificar ampollas de vidrio de $1 \mathrm{ml}$ que fueron posteriormente cerradas y sometidas a las mismas condiciones de almacenamiento que los envases de ese mismo lote. La solución en las ampollas constituyó una referencia a la hora de estudiar la estabilidad de esa misma solución en los demás envases.

\section{Cromatografía líquido-líquido de alta resolución (HPLC)}

En el estudio de la evolución del contenido en hidroxocobalamina de las soluciones bajo ensayo en función del tiempo a diferentes temperaturas, así como en la caracterización de la materia prima, esta técnica analítica ha sido esencial. La aparición de un solo pico correspondiente a hidroxocobalamina en el desarrollo del cromatograma de cada muestra, fue concluyente en la determinación de la estabilidad. Así mismo lo es la ausencia de picos secundarios correspondientes a productos de descomposición por inestabilidad del antídoto en solución.

Se ha utilizado un cromatógrafo de la firma PerkinElmer equipado con una bomba binaria modelo Flexar Binary LC Pump y un inyector Kit-Flexar Manual $20 \mathrm{ml}$. La detección se llevó a cabo con un detector espectrofotométrico Flexar UVIVIS a longitud de onda fija de $351 \mathrm{~nm}$, correspondiente al máximo de absorción del cloruro de hidroxocobalamina. El software utilizado para procesar los datos cromatográficos fue el Chromera Software KIT V3.0.

Tomando como referencia la técnica analítica para "Sustancias relacionadas" especificada en la Farmacopea Europea

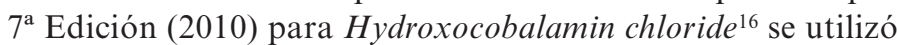
una columna cromatográfica con fase estacionaria $\mathrm{C} 18$ de PerkinElmer modelo Col-Analytical C18 $5 \mathrm{~mm} 150 \times 4.6 \mathrm{~mm}$ (Ref. N9303513). Como fase móvil se empleó una mezcla con 25 volúmenes de metanol y 75 volúmenes de una solución con $15 \mathrm{~g} / 1$ de ácido cítrico y 8,1 g/1 de fosfato bisódico. La veloci- dad de flujo de la fase móvil fue de $1,5 \mathrm{ml} / \mathrm{min}$. El tiempo total especificado para el desarrollo del cromatograma fue al menos cuatro veces el tiempo de retención de la hidroxocobalamina. Las muestras fueron diluidas con fase móvil a una concentración entorno a $0,05 \mathrm{mg} / \mathrm{ml}$ y pinchadas en el cromatógrafo.

\section{Espectrofotometría infrarroja}

La caracterización de los materiales de partida se ha llevado a cabo, entre otras técnicas, mediante espectrofotometría de infrarrojos tanto por transmisión como por reflexión. Estas técnicas se han empleado también para estudiar la evolución de la estabilidad de las muestras bajo ensayo, mediante comparación del espectro de referencia de la hidroxocobalamina con el de la muestra bajo estudio desecada. El software del equipo Spectra $I R$ permite la obtención de un coeficiente de correlación que expresa el grado de semejanza entre ambos espectros.

Se ha empleado un espectrofotómetro IR-FT Paragón1000 de PerkinElmer. Para las determinaciones por reflexión se ha utilizado el accesorio Gladia ATR de PerkinElmer acoplado al espectrofotómetro.

\section{Espectrofotometría ultravioleta-visible (UV/V)}

El estudio de los barridos espectrofotométricos ultravioletavisible de las soluciones bajo ensayo, el control de las longitudes de onda de máxima absorción, y los valores de absorbancia de las diluciones efectuadas, ha sido también esencial para el estudio de la estabilidad. Se ha utilizado un espectrofotómetro ultravioleta-visible Lambda 25 de la firma PerkinElmer, con el software Spectra $U V / V$ para el tratamiento de los resultados. Como diluyente para la preparación de las muestras se empleó ácido clorhídrico 0,01 N. El rango de longitudes de onda para los barridos espectrales fue de 350 a $200 \mathrm{~nm}$. Las medidas de absorbancia para la cuantificación de las muestras se realizaron a $351 \mathrm{~nm}$, longitud de onda de máxima absorción para hidroxocobalamina. El contenido teórico de las soluciones preparadas fue de 1 miligramo en 40 microlitros.

\section{Determinaciones de pH}

El pH de las muestras bajo estudio se ha determinado por inmersión del electrodo en un tubo de ensayo con diámetro interior ajustado al diámetro del electrodo, de forma que el volumen de muestra necesario fuera mínimo. Se ha utilizado un $\mathrm{pH}$-metro Crison PH 25 con electrodo ácido-base en medio acuoso.

\section{Ensayo de esterilidad mediante filtración por membrana}

La esterilidad de las soluciones preparadas por vía aséptica fue verificada para cada lote mediante el sistema Steritest $^{R}$ de la firma Millipore $^{R}$, ensayo que está normalizado para su realización mediante filtración por membrana. Los medios de cultivo 


\section{Estabilidad de la hidroxocobalamina en agua para inyección como antídoto contra cianuros...}

utilizados para la realización del ensayo fueron: TSA (Trypcase Soja Agar) para bacterias aerobias y Tioglicolato para bacterias anaerobias. La incubación se llevó a cabo a $35^{\circ} \mathrm{C}$ durante 7 días. La presencia o ausencia de crecimiento bacteriano se detecta por enturbiamiento del medio de cultivo

\section{Ensayo de ausencia de endotoxinas bacterianas ("pirógenos")}

Se llevo a cabo mediante el test enzimático LAL (Lymulus Amebocyte Lysate). El reactivo utilizado es de la firma $L o n z a^{R}$ : Pyrogent Plus Single Test Kit 24 T N-189-125. Se verificó la idoneidad del ensayo mediante la utilización de controles de endotoxina positivos.

\section{Método de almacenamiento y programa de estudio}

El diseño de los estudios de estabilidad está basado en la información y propiedades de la hidroxocobalamina. Incluye el estudio de los atributos susceptibles de cambio durante el almacenamiento y que pueden afectar a la calidad, seguridad o eficacia del preparado. Debe cubrir los atributos físicos, químicos, biológicos y microbiológicos ${ }^{19}$. Dadas las características de los envases para soluciones parenterales, en las condiciones de almacenamiento no es aplicable el control de la humedad relativa del aire. Las temperaturas de almacenamiento de las muestras para el estudio de su estabilidad en función del tiempo fueron: $4^{\circ}$ $\mathrm{C}$ (refrigerador), $25^{\circ} \mathrm{C}$ (temperatura ambiente) y $37^{\circ} \mathrm{C}$ (estufa). No procede la realización de ensayos de fotosensibilidad, pues el preparado se debe conservar en su envase de origen protegido de la luz. En todos los casos las muestras se mantuvieron protegidas de la luz.

Aunque para cada muestra se especifican los días exactos en los que cada determinación se llevó a cabo, en general, los tiempos de análisis fueron a los 0, 3, 6 y 12 meses.

\section{Lotes de prueba realizados}

La composición de la solución inyectable para los cuatro lotes preparados fue la siguiente:

- Cloruro de hidroxocobalamina - $25 \mathrm{mg}$

- Agua para inyección c.s.p. - $1 \mathrm{ml}$

\section{Lote número 1.}

Fecha de preparación: 02.12.2009

Tipo de envase utilizado: Ampollas.

\section{Lote número 2.}

Fecha de preparación: 17.12.2009

Tipo de envases utilizado: Ampollas y bolsas de polipropileno.

La especificación "tapón nuevo" y "tapón inicial" diferencia dos sublotes dentro de este lote. En el sublote "tapón nuevo" las bolsas fueron cerradas con tapones tal y como se reciben del fabricante ("tapón nuevo"). En el sublote "tapón inicial" los tapones fueron sometidos a un proceso de lavado y autoclavado previo a su utilización ("tapón inicial"). El objetivo de esta diferenciación fue verificar la ausencia de un proceso de cesión (leaching) por parte del tapón sin tratamiento. Los resultados no han revelado diferencias entre ambos sublotes.

\section{Lote número 3.}

Fecha de preparación: 04.03.2010

Envases utilizados: Ampollas y viales COC.

\section{Lote número 4.}

Fecha de preparación: 16.12.2010

Envases utilizados: Ampollas y viales de polipropileno.

Los tapones utilizados para cerrar los viales fueron empleados según se recibieron del fabricante: limpios, estériles y apirógenos. No se realizó lavado ni autoclavado de los mismos, según se ha explicado para un sublote de bolsas.

\section{RESULTADOS}

En la Tabla 1 se exponen los resultados obtenidos mediante espectrofotometría ultravioleta y visible del estudio de estabilidad de la solución inyectable de hidroxocobalamina como antídoto contra cianuros. Se expone la evolución del contenido en cloruro de hidroxocobalamina (expresado en porcentaje del contenido teórico o nominal) en función del tiempo (expresado en días de ensayo). Se han estudiado cuatro lotes, todos ellos con la misma composición de la solución inyectable. A cada lote corresponde un envase farmacéutico diferente (ampollas, bolsas y dos tipos de viales), de los que se han sometido muestras a diferentes temperaturas de conservación $\left(4^{\circ} \mathrm{C}, 25^{\circ} \mathrm{C}\right.$ y $37^{\circ} \mathrm{C}$, según cada caso) para determinar su estabilidad en el tiempo.

Para cada muestra, la determinación del contenido se ha realizado en el tiempo cero, correspondiente a la fecha de preparación del lote, y a los 3, 6 y 12 meses aproximadamente, según la muestra. Para cada punto se han expresado los días de ensayo transcurridos.

Se ha calculado la media estadística de los valores de contenido obtenidos en los diferentes tiempos para cada lote, y se expone su variabilidad expresada en función de la desviación estándar, como el rango de contenido en hidroxocobalamina para un intervalo de confianza del 95 por ciento $(\alpha=0,05)$. Cuanto más baja es la variabilidad de la media estadística del contenido en función del tiempo más estable es la solución estudiada a la temperatura y en el envase de ensayo.

Se especifica también el "Tipo de Envase" en que se ha realizado el estudio, según fueran ampollas de vidrio, bolsas de polipropileno (PP), viales de un compuesto olefínico complejo (COC) o viales de polipropileno (PP).

El Lote $\mathrm{N}^{\mathrm{o}} 1$ ha sido estudiado solo en ampollas de vidrio de primera clase hidrolítica. Por las excelentes propiedades e inercia del vidrio como envase farmacéutico, la evolución del contenido en hidroxocobalamina de la solución envasada en ampollas se ha tomado como referencia respecto a la evolución en los restantes envases estudiados.

La representación gráfica de los resultados expuestos en la Tabla 1 para el contenido en hidroxocobalamina en función del 
Tabla 1. Evolución del contenido en hidroxocobalamina (espectrofotometría ultravioleta y visible).

\begin{tabular}{|c|c|c|c|c|c|c|c|}
\hline $\begin{array}{c}\text { Temperatura } \\
\text { De ensayo }\end{array}$ & $\begin{array}{l}\text { Tipo de } \\
\text { envase }\end{array}$ & Lote & $\begin{array}{c}\text { Días de } \\
\text { ensayo }\end{array}$ & $\begin{array}{c}\text { Contenido } \\
\text { (\%HCB) }\end{array}$ & Media & $\begin{array}{l}\text { Variabilidad } \\
p=0,95( \pm)\end{array}$ & $\mathbf{n}$ \\
\hline \multirow{28}{*}{$4^{\circ} \mathrm{C}$} & \multirow{14}{*}{ Ampollas } & \multirow{4}{*}{$\mathrm{N}^{\circ} 1$} & 0 & 98,84 & \multirow{4}{*}{99,44} & \multirow{4}{*}{0,88} & \\
\hline & & & 104 & 100,1 & & & 4 \\
\hline & & & 199 & 99,32 & & & 4 \\
\hline & & & 419 & 99,51 & & & \\
\hline & & & 0 & 99,43 & & & \\
\hline & & No 2 & 89 & 100,72 & & & \\
\hline & & $N 2$ & 184 & 101,52 & 99,93 & 2,59 & 4 \\
\hline & & & 353 & 98,04 & & & \\
\hline & & & 0 & 100,46 & & & \\
\hline & & $\mathrm{N}^{\circ} 3$ & 107 & 100,18 & 100,46 & 0,45 & 3 \\
\hline & & & 276 & 100,74 & & & \\
\hline & & & 0 & 100,09 & & & \\
\hline & & $\mathrm{N}^{\circ} 4$ & 117 & 102,46 & 101,73 & 2,27 & 2 \\
\hline & & & 276 & 102,63 & & & \\
\hline & & & 0 & 99,43 & & & \\
\hline & & & 89 & 100,76 & & & \\
\hline & Bolsas t.1. & $\mathrm{N}^{0} 2$ & 184 & 102,56 & 100,55 & 2,51 & 4 \\
\hline & & & 353 & 99,44 & & & \\
\hline & & & 0 & 99,43 & & & \\
\hline & Bolsas & & 89 & 101,85 & & & \\
\hline & t.n. & $N 2$ & 184 & 102,62 & 101,51 & 5,09 & 4 \\
\hline & & & 353 & 99,13 & & & \\
\hline & & & 0 & 102,09 & & & \\
\hline & Viales & $\mathrm{N}^{\circ} 3$ & 107 & 102,38 & 102,09 & 0,47 & 3 \\
\hline & & & 276 & 101,79 & & & \\
\hline & & & 0 & 100,09 & & & \\
\hline & Viales PP & $\mathrm{N}^{\circ} 4$ & 117 & 102,46 & 101,64 & 2,16 & 3 \\
\hline & & & 276 & 102,36 & & & \\
\hline & & & 0 & 99,43 & & & \\
\hline & & No ? & 89 & 99,62 & & & 4 \\
\hline & & $N 2$ & 184 & 99,59 & 99,62 & 0,28 & 4 \\
\hline & & & 353 & 99,83 & & & \\
\hline & Amnollas & & 0 & 99,62 & & & \\
\hline & Ampoilas & $\mathrm{N}^{\circ} 3$ & 107 & 99,59 & 99,68 & 0,21 & 3 \\
\hline & & & 276 & 99,83 & & & \\
\hline & & & 0 & 100,09 & & & \\
\hline & & $\mathrm{N}^{\circ} 4$ & 117 & 103,44 & 101,73 & 2,68 & 3 \\
\hline & & & 276 & 101,66 & & & \\
\hline $25^{\circ} \mathrm{C}$ & & & 0 & 99,43 & & & \\
\hline & Bolsas t i & $\mathrm{N}^{\circ} 2$ & 89 & 99,66 & 10138 & 477 & 4 \\
\hline & Bolsas t.1. & $N 2$ & 184 & 100,94 & 101,38 & $4, / 1$ & 4 \\
\hline & & & 353 & 105,47 & & & \\
\hline & & & 0 & 99,43 & & & \\
\hline & Bolsas & $\mathrm{N}^{\circ} 2$ & 89 & 99,03 & 10083 & 379 & 4 \\
\hline & t.n. & 102 & 184 & 100,9 & 100,05 & 5,19 & 4 \\
\hline & & & 353 & 103,95 & & & \\
\hline & & & 0 & 100,09 & & & \\
\hline & Viales PP & $\mathrm{N}^{\circ} 4$ & 117 & 103,56 & 101,91 & 2,79 & 3 \\
\hline & & & 276 & 102,07 & & & \\
\hline & & & 0 & 98,84 & & & \\
\hline & & $\mathrm{N}^{\circ} 1$ & 60 & 98,41 & 9820 & 331 & 4 \\
\hline & & $\mathbf{N} 1$ & 199 & 100,08 & 98,20 & 3,31 & 4 \\
\hline & & & 368 & 95,47 & & & \\
\hline & & & 0 & 99,43 & & & \\
\hline & & & 45 & 99,64 & & & \\
\hline & & $\mathrm{N}^{\circ} 2$ & 89 & 99,51 & 99,68 & 4,22 & 5 \\
\hline & Ampollas & & 184 & 103,31 & & & \\
\hline & & & 353 & 96,53 & & & \\
\hline & & & 0 & 100,52 & & & \\
\hline & & $\mathrm{N}^{\circ} 3$ & 107 & 102 & 100,38 & 2,71 & 3 \\
\hline & & & 276 & 98,62 & & & \\
\hline & & & 0 & 100,09 & & & \\
\hline & & $\mathrm{N}^{\circ} 4$ & 117 & 102,07 & 101,24 & 1,65 & 3 \\
\hline & & & 276 & 101,56 & & & \\
\hline $37^{\circ} \mathrm{C}$ & & & 0 & 99,43 & & & \\
\hline & & & 45 & 100,75 & & & \\
\hline & Bolsas t.i. & $\mathrm{N}^{\circ} 2$ & 89 & 105,61 & 113,29 & 30,85 & 5 \\
\hline & & & 184 & 119,15 & & & \\
\hline & & & 353 & 141,51 & & & \\
\hline & & & 0 & 99,43 & & & \\
\hline & & & 45 & 99,84 & & & \\
\hline & Bolsas & $\mathrm{N}^{\circ} 2$ & 89 & 107,7 & 114,17 & 34,59 & 5 \\
\hline & & & 184 & 116,77 & & & \\
\hline & & & 353 & 147,13 & & & \\
\hline & & & 0 & 102,03 & & & \\
\hline & Viales & $\mathrm{N}^{\circ} 3$ & 107 & 102,26 & 102,35 & 0,60 & 3 \\
\hline & $c 00$ & & 276 & 102,76 & & & \\
\hline & & & 0 & 100,09 & & & \\
\hline & Viales PP & $\mathrm{N}^{\circ} 4$ & 117 & 102,28 & 101,19 & 1,75 & 3 \\
\hline & & & 276 & 101,19 & & & \\
\hline
\end{tabular}

tiempo de ensayo para cada tipo de envase estudiado, se expone en las Gráficas 1,2 y 3 , a $4^{\circ}, 25^{\circ}$ y $37^{\circ} \mathrm{C}$, respectivamente.

Se significa aquí que el rango de conformidad especificado por la Farmacopea de Estados Unidos para $\mathrm{Hi}$ droxocobalamin Injection respecto al "contenido etiquetado" o contenido teórico de la solución inyectable oscila entre un valor mínimo del 95 por ciento y un máximo del 115 por ciento ${ }^{15}$.

Para simplificar la representación gráfica de los resultados obtenidos, y dado el agrupamiento de los mismos, se han calculado los valores medios del contenido en hidroxocobalamina obtenidos para las ampollas de los cuatro lotes estudiados. Los valores medios obtenidos para el contenido en hidroxocobalamina junto al intervalo de variabilidad para una confianza del 95 por ciento se exponen en la Tabla 2. En las gráficas 1, 2 y 3 se han representado en trazo continuo y en color azul estos resultados medios para la solución envasada en "Ampollas".

Los resultados obtenidos muestran que el contenido $(\%)$ en hidroxocobalamina de las soluciones envasadas en ampollas permanece uniforme, dentro del rango de aceptabilidad especificado, y con muy baja variabilidad (Tabla 2).

La solución preparada para el Lote $\mathrm{N}^{\circ} 2$ se envasó, además de en ampollas, en envases (bolsas) de polipropileno de $100 \mathrm{ml}$. Tal y como se ha expuesto en el apartado Material y Métodos, se han diferenciado dos sublotes según el tratamiento dado a los tapones utilizados para cerrar las bolsas. No obstante, los resultados obtenidos demuestran que no existen diferencias significativas entre ambos sublotes, en cualquiera de las condiciones de ensayo estudiadas. Por ello y para simplificar la representación gráfica de resultados, en las Gráficas 1, 2 y 3 sólo se han representado en trazo discontinuo y color verde los resultados para las bolsas con tapón nuevo (Bolsas PP) y no se han representado para las bolsas cerradas con el tapón tratado, denominadas "bolsas con tapón inicial".

La solución preparada para el Lote $\mathrm{N}^{\mathrm{o}} 3$ se envasó en ampollas y en viales del material plástico denominado COC. El contenido en hidroxocobalamina de sus soluciones ha permanecido invariable, según se deduce del estrecho intervalo de valores para la media para un intervalo de confianza del 95 por ciento $(102,09 \%$ $\pm 0,47 \%$, a $4^{\circ} \mathrm{C} ; 102,35 \% \pm 0,60 \%$, a $\left.37^{\circ} \mathrm{C}\right)$ y de la representación gráfica de sus resultados, en línea de trazos y puntos en color rojo. Los resultados obtenidos permiten afirmar que los viales COC constituyen un envase adecuado para disponer de soluciones estables de hidroxocobalamina inyectable. El inconveniente de estos envases, como se verá en el apartado Discusión, es estético pues estos viales no ocultan el intenso color rojo de las soluciones de hidroxocobalamina a la alta concentración necesaria para actuar como antídoto contra cianuros.

La solución preparada para el Lote $\mathrm{N}^{\circ} 4$ se envasó también en ampollas, y en viales de polipropileno (Viales 
Estabilidad de la hidroxocobalamina en agua para inyección como antídoto contra cianuros...

Tabla 2. Valores medios del contenido en hidroxocobalamina para los cuatro lotes de ampollas preparados.

\begin{tabular}{|c|c|c|c|c|c|c|c|c|c|}
\hline \multirow[b]{2}{*}{ Días } & \multicolumn{3}{|c|}{$\begin{array}{c}\text { Temperatura de } \\
\text { ensayo } 4^{\circ} \mathrm{C}\end{array}$} & \multicolumn{3}{|c|}{$\begin{array}{c}\text { Temperatura de } \\
\text { ensayo } 25^{\circ} \mathrm{C}\end{array}$} & \multicolumn{3}{|c|}{$\begin{array}{c}\text { Temperatura de } \\
\text { ensayo } 37^{\circ} \mathrm{C}\end{array}$} \\
\hline & Contenido & Intervalo $( \pm)$ & $\mathrm{n}$ & Contenido & Intervalo $( \pm)$ & $\mathrm{n}$ & Contenido & Intervalo $( \pm)$ & $\mathrm{n}$ \\
\hline 0 & 99,71 & 1,22 & $\mathrm{n}=4$ & 99,71 & 0,54 & $\mathrm{n}=3$ & 99,72 & 1,25 & $\mathrm{n}=4$ \\
\hline 90 & 100,87 & 1,86 & $\mathrm{n}=4$ & 100,88 & 3,54 & $\mathrm{n}=3$ & 100,50 & 3,11 & $\mathrm{n}=4$ \\
\hline 180 & 100,42 & 2,16 & $\mathrm{n}=2$ & 99,59 & & $\mathrm{n}=1$ & 101,70 & 3,17 & $\mathrm{n}=2$ \\
\hline 270 & 101,69 & 1,85 & $\mathrm{n}=2$ & 100,75 & 1,79 & $\mathrm{n}=2$ & 100,09 & 2,88 & $\mathrm{n}=2$ \\
\hline 360 & 98,78 & 1,44 & $\mathrm{n}=2$ & 99,83 & & $\mathrm{n}=1$ & 96,00 & 1,04 & $\mathrm{n}=2$ \\
\hline
\end{tabular}

PP). Aunque su material constitutivo es idéntico al de las bolsas empleadas para la fabricación de sueros, su mayor espesor de pared le confiere más rigidez y mejores propiedades barrera. Se han utilizado viales de color ámbar por ocultar mejor el intenso color de estas soluciones. Estéticamente es el envase más apropiado. Los resultados obtenidos, representados en las Gráficas 1, 2 y 3 mediante un trazo discontinuo en color celeste, les presentan como una buena alternativa a los Viales COC a los que igualan en cuanto a estabilidad de la solución y superan estéticamente por ocultar su aspecto desfavorable.

En la Tabla 3 se muestran los resultados obtenidos mediante cromatografía líquido - líquido de alta resolución (HPLC) sobre muestras conservadas a $4^{\circ}$ y $37^{\circ} \mathrm{C}$, y para cada tipo de envase (ampollas, bolsas de polipropileno, viales de COC o viales de polipropileno).

El contenido en hidroxocobalamina expresado en porcentaje respecto al valor teórico o nominal, así como la aparición o no en el cromatograma de picos secundarios resultantes de la degradación del principio activo, proporciona información valiosa para concluir sobre la estabilidad de esa muestra. La no aparición de picos secundarios es un dato concluyente para confirmar su estabilidad.

El tiempo de retención para el pico de hidroxocobalamina obtenido en los cromatogramas mediante la técnica utilizada fue de 2,860 minutos ( $\pm 0,169$ minutos; $\mathrm{p}=0,95 ; \mathrm{n}=15$ ).

Los resultados de la Tabla 3 muestran la estabilidad a $4^{\circ} \mathrm{C}$ de todos los lotes y envases estudiados, en algunos casos durante un

Tabla 3. Evolución del contenido en hidroxocobalamina (cromatografía líquido-líquido de alta resolución).

\begin{tabular}{|cccccc|}
\hline $\begin{array}{c}\text { Temperatura } \\
\text { de } \\
\text { ensayo }\end{array}$ & $\begin{array}{c}\text { Tipo } \\
\text { de } \\
\text { Envase }\end{array}$ & Lote & $\begin{array}{c}\text { Días } \\
\text { de ensayo }\end{array}$ & $\begin{array}{c}\text { Contenido } \\
\mathbf{( \% H C B )}\end{array}$ & $\begin{array}{c}\text { Tiempo } \\
\text { de } \\
\text { retención }\end{array}$ \\
\hline $4^{\circ} \mathrm{C}$ & Ampollas & 1 & 472 & 100,00 & 2,952 \\
$4^{\circ} \mathrm{C}$ & Ampollas & 2 & 457 & 100,00 & 2,941 \\
$4^{\circ} \mathrm{C}$ & Ampollas & 3 & 385 & 100,00 & 2,919 \\
$4^{\circ} \mathrm{C}$ & Ampollas & 4 & 99 & 100,00 & 2,815 \\
$4^{\circ} \mathrm{C}$ & Viales COC & 3 & 387 & 100,00 & 2,825 \\
$4^{\circ} \mathrm{C}$ & Viales PP & 4 & 105 & 99,00 & 2,815 \\
$4^{\circ} \mathrm{C}$ & Bolsas PP & 2 & 464 & 99,67 & 2,792 \\
$37^{\circ} \mathrm{C}$ & Ampollas & 1 & 483 & 96,42 & 2,986 \\
$37^{\circ} \mathrm{C}$ & Ampollas & 2 & 474 & 90,12 & 2,660 \\
$37^{\circ} \mathrm{C}$ & Ampollas & 3 & 397 & 89,46 & 2,791 \\
$37^{\circ} \mathrm{C}$ & Ampollas & 4 & 115 & 95,46 & 2,832 \\
$37^{\circ} \mathrm{C}$ & Bolsa t.i. & 2 & 474 & 97,13 & 2,799 \\
$37^{\circ} \mathrm{C}$ & Viales COC & 3 & 397 & 97,85 & 2,985 \\
$37^{\circ} \mathrm{C}$ & Viales PP & 4 & 115 & 97,48 & 2,911 \\
$115^{\circ} \mathrm{C}$ & Ampollas & 4 & Ver & 86,59 & 2,879 \\
\hline
\end{tabular}

total de 472 días ( 15 meses). A esta temperatura, el contenido en hidroxocobalamina permaneció prácticamente invariable (media $=99,81 \% \pm 0,69 \% ; p=0,95 ; n=7)$. La pequeña oscilación en el porcentaje del contenido sobre el valor teórico (25 miligramos de cloruro de hidroxocobalamina por mililitro) se debe a la variabilidad inherente al método analítico empleado. Estos resultados se confirman con los cromatogramas de cada muestra, donde se aprecia un único pico principal de hidroxocobalamina, y ausencia total de picos secundarios de degradación. Estos resultados concuerdan con los obtenidos en las determinaciones espectrofotométricas y permiten afirmar que la solución de hidroxocobalamina es estable a $4^{\circ} \mathrm{C}$ durante un periodo superior a 15 meses.

La homogeneidad en los resultados obtenidos para las ampollas de los cuatro lotes a $4^{\circ} \mathrm{C}$ las valida como referencia frente a otras muestras sometidas a un estrés térmico superior.

Cuando las ampollas de los cuatro lotes preparados se conservaron a $37^{\circ} \mathrm{C}$ durante el mismo periodo de tiempo, el porcentaje de hidroxocobalamina fue ligeramente inferior $($ media $=$ $92,87 \% \pm 6,08 \% ; \mathrm{p}=0,95 ; \mathrm{n}=4)$. Fue del $98 \%$ a los 9 meses y su disminución fue inapreciable a los 3 meses.

En todos los casos, el contenido en hidroxocobalamina expresado en porcentaje respeto al valor teórico y determinado espectrofotométricamente, se mantuvo dentro del rango de aceptación especificado en la farmacopea (95\%-115\%).

Los cromatogramas obtenidos de muestras de ampollas mantenidas durante más de 15 meses a $37^{\circ} \mathrm{C}$ mostraron un pequeño pico secundario de degradación, con un tiempo de retención medio de 3,791 minutos ( $\pm 0,293$ minutos; $p=0,95$; $\mathrm{n}=8)$.

La cromatografía líquido-líquido se ha mostrado en este estudio sobre la estabilidad de las soluciones de cloruro de hidroxocobalamina como antídoto contra cianuros, como una técnica más resolutiva para la detección de diferencias y productos de degradación. Frente a estos últimos resultados expuestos, los valores de longitud de onda de máxima absorción obtenidos en las determinaciones espectrofotométricas ultravioleta-visible permanecen prácticamente invariables aun cuando la aparición de picos secundarios ocurre en mayor extensión: A $37^{\circ} \mathrm{C}$ el valor medio de la longitud de onda de máxima absorción es de 350,66 $\mathrm{nm}( \pm 0,36 \mathrm{~nm} ; \mathrm{p}=0,95 ; \mathrm{n}=25)$ frente a $350,64 \mathrm{~nm}( \pm 0,23 \mathrm{~nm}$; $\mathrm{p}=0,95 ; \mathrm{n}=22)$ a $4^{\circ} \mathrm{C}$.

Los resultados obtenidos de los cromatogramas realizados sobre las muestras de ampollas a $37^{\circ} \mathrm{C}$ de los lotes 3 y 4 (Tabla 3) almacenadas durante 13 meses y 4 meses respectivamente, muestran el aumento del pico secundario a expensas de la concentración de cloruro de HCB. A los cuatro meses el pico secundario es prácticamente inapreciable. 


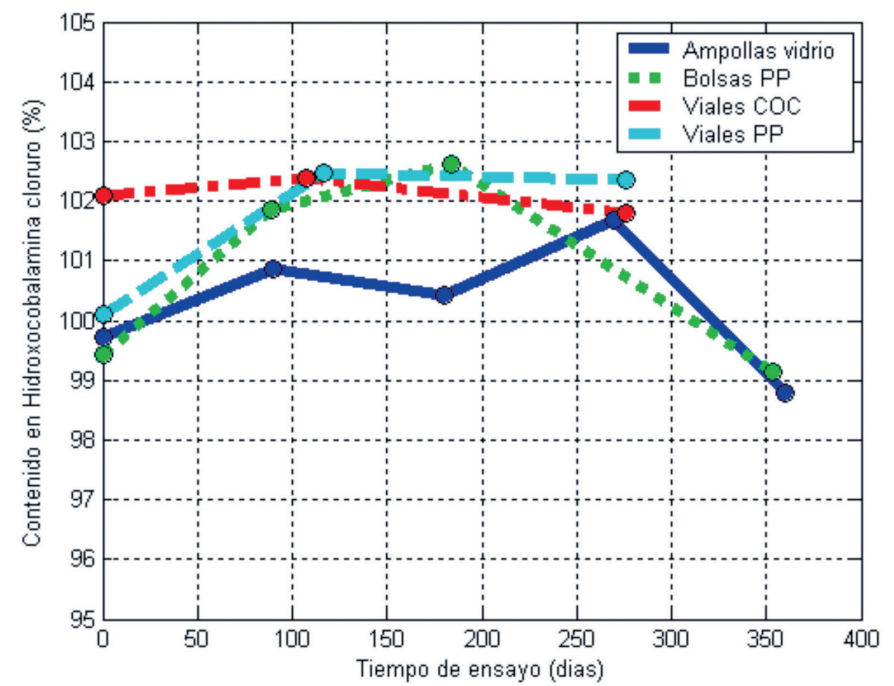

Figura 1. Estabilidad de las soluciones de Hidroxicobalamina cloruro a $4^{\circ} \mathrm{C}$.

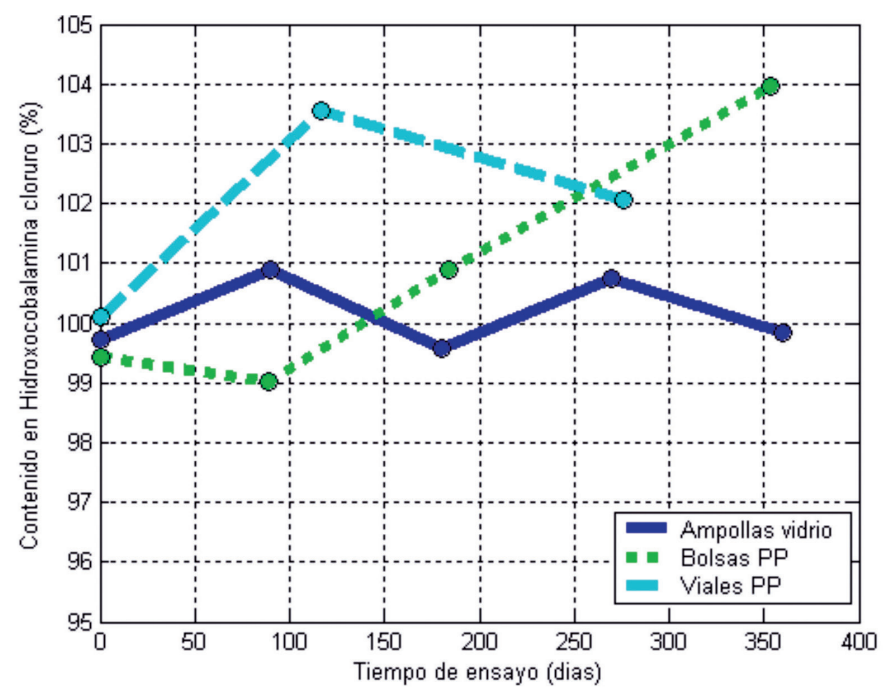

Figura 2. Estabilidad de las soluciones de Hidroxicobalamina cloruro a $25^{\circ} \mathrm{C}$.

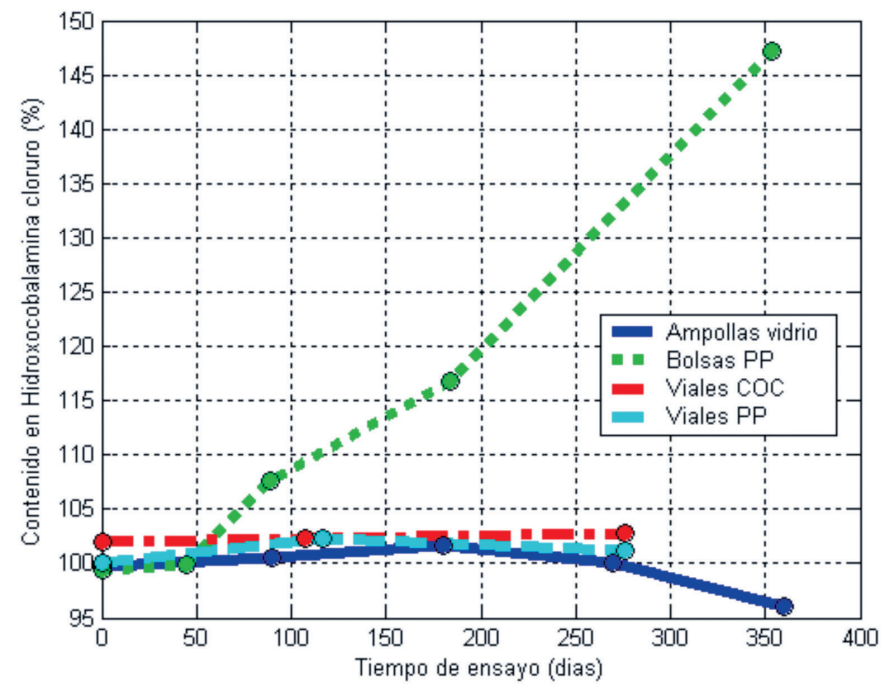

Figura 3. Estabilidad de las soluciones de Hidroxicobalamina cloruro a $37^{\circ} \mathrm{C}$.
Aunque $37^{\circ} \mathrm{C}$ no es temperatura adecuada de almacenamiento para ningún medicamento, en este estudio se ha utilizado para conseguir condiciones de estrés que provoquen un envejecimiento acelerado de la solución bajo estudio y su interacción con el envase, si hubiera lugar.

A $25^{\circ} \mathrm{C}$ las soluciones permanecieron inalteradas, pues no aparecen picos secundarios de degradación en el desarrollo de los cromatogramas.

En las bolsas de polipropileno sometidas a $37^{\circ} \mathrm{C}$ (Tabla 1, lote $\mathrm{N}^{\mathrm{o}} 2$ ) se produce una concentración de la solución envasada por pérdida de agua a través del envase. Después de 12 meses, el contenido en hidroxocobalamina determinado por espectrofotometría ultravioleta/visible fue superior al 140 por ciento de la cantidad nominal. Este efecto de concentración hace que la proporción relativa del pico secundario en el cromatograma sea inferior. A $25^{\circ} \mathrm{C}$ el efecto de concentración ocurre en mucha menor extensión (entre el 104\% y el 105,5\%).

Por lo anterior, las bolsas de polipropileno no son un envase adecuado para esta solución, a menos que se conserven a temperaturas inferiores a $25^{\circ} \mathrm{C}$ durante todo el periodo de vida útil del medicamento.

Tanto los viales de polipropileno como los viales de COC (Copolímero Olefínico Complejo) presentaron un buen comportamiento como envases farmacéuticos primarios para la solución parenteral del antídoto que se estudia. La solución fue estable y el envase impermeable, según los resultados que se exponen en la Tabla 1 para los lotes 3 y 4 . En la Tabla 2 se exponen los resultados obtenidos mediante cromatografía para ambos tipos de viales, tanto a $4^{\circ} \mathrm{C}$ como a $37^{\circ} \mathrm{C}$. A $4^{\circ} \mathrm{C}$ el contenido en hidroxocobalamina permanece invariable, y a $37^{\circ} \mathrm{C}$ la reducción es insignificante. En el cromatograma obtenido para esta última muestra, el pico secundario por estrés térmico de la solución de hidroxocobalamina es prácticamente inapreciable.

Finalmente, se analizaron mediante HPLC muestras de solución envasadas en ampollas que habían sido autoclavadas a $115^{\circ}$ C durante 30 minutos, el mismo día de su preparación. De los resultados obtenidos, en la Tabla 2 se expone solo el correspondiente al lote número 4 , por ser idéntico a los que presentaron los tres lotes restantes tratados en las mismas condiciones. A diferencia de los resultados obtenidos por almacenamiento prolongado a $37^{\circ} \mathrm{C}$, la esterilización en autoclave provoca la aparición de dos nuevos picos secundarios, uno anterior al pico principal de hidroxocobalamina, con un tiempo de retención 2,418 minutos $( \pm 0,015$ minutos; $\mathrm{p}=0,95 ; \mathrm{n}=4)$ y otro posterior al de hidroxocobalamina, con tiempo de retención 3,844 minutos ( \pm $0,030$ minutos; $\mathrm{p}=0,95 ; \mathrm{n}=4)$. El porcentaje de hidroxocobalamina se reduce al 86,59 por ciento $(79,85 \%, 78,12 \%$ y $79,73 \%$ para los lotes 1, 2 y 3, respectivamente) quedando en todos los casos fuera del rango de conformidad especificado (95-115\%).

Estos resultados conducen a la conclusión de que la solución inyectable de hidroxocobalamina no puede ser esterilizada por calor sino que deberá ser tratada mediante filtración esterilizante, y su proceso de fabricación llevado a cabo por vía aséptica sin esterilización terminal.

Los espectros infrarrojos realizados sobre muestras desecadas de los diferentes lotes y los coeficientes de semejanza o correlación respecto a la sustancia de referencia permiten confirmar los resultados de estabilidad mencionados. 


\section{Estabilidad de la hidroxocobalamina en agua para inyección como antídoto contra cianuros...}

Los valores de $\mathrm{pH}$ medidos para cada muestra permanecieron invariables respecto a los valores iniciales. Esto es aplicable a cualquier lote, tiempo y condición de almacenamiento.

La esterilidad de la solución preparada de cada lote se comprobó mediante el ensayo de filtración por membrana (Steritest ${ }^{R}$ de Millipore).

La solución preparada de cada lote cumplió el ensayo de endotoxinas bacterianas llevado a cabo mediante el test enzimático LAL (Lymulus Amebocyte Lysate). El contenido fue inferior a $0,125 \mathrm{UE} / \mathrm{ml}$ (límite de sensibilidad del reactivo). Las soluciones fueron apirógenas.

Finalmente, ninguna de las muestras estudiadas presentó cambios físicos evidentes: cambios de color, precipitación, aspecto o cualquier otro.

\section{DISCUSIÓN}

De los diferentes antídotos contra cianuros disponibles, la combinación tiosulfato sódico y nitrito sódico ha sido tradicionalmente de elección como antídotos primarios para el tratamiento de la intoxicación por cianuros en Defensa N.B.Q. ${ }^{5,13}$. Sin entrar en la consideración de factores como la eficacia y la seguridad farmacológica, motivos tales como la más fácil disponibilidad de los principios activos, inferior coste, mayor estabilidad y periodo de caducidad, e incomparable relación coste/ eficacia, decantaron claramente la decisión. Además, el importe necesario para la renovación de un stock inmovilizado como el necesario para la constitución y mantenimiento de una reserva farmacéutica estratégica nacional, es otro importante factor a favor.

Alternativamente, la combinación tiosulfato sódico e hidroxocobalamina ha sido presentada más recientemente por su mejor relación beneficio-riesgo, especialmente cuando la intoxicación por cianuros se produce a la vez que con monóxido de carbono ${ }^{1,6,14}$. En estos casos, aunque con las dosis normales recomendadas de nitrito sódico el pico máximo de metahemoglobina se presenta a los 50 minutos de la administración y la reducción en la capacidad de transporte de oxígeno no es nunca superior al 21 por ciento ${ }^{19}$, la hidroxocobalamina es más segura. Por ello se ha considerado de interés disponer de una solución inyectable de hidroxocobalamina para su incorporación al arsenal terapéutico necesario en las unidades de emergencias y en las células de estabilización con soporte vital avanzado, como antídoto en los síndromes de intoxicación concomitante por cianuros y monóxido de carbono por inhalación de humos en incendios en espacios cerrados.

En el presente trabajo se ha estudiado la estabilidad de la hidroxocobalamina en solución inyectable en agua para inyección. Su indicación como antídoto contra cianuros tiene varias connotaciones. En primer lugar, la dosis es 2.500 veces superior a la dosis recomendada por su actividad sobre la hematopoyesis y la anemia perniciosa, de alrededor de 1 miligramo por mililitro $^{20}$, y el contenido en hidroxocobalamina de la solución inyectable como antídoto es 25 veces superior. Por definición de la monografía oficial de la United States Pharmacopeia (U.S.P.) la inyección de hidroxocobalamina es una solución estéril de hidroxocobalamina en agua para inyección ${ }^{15}$.
En segundo lugar, la forma de presentación de cualquier antídoto debe ser adecuada para su rápida administración en situaciones de urgencia. Es por ello que se ha estudiado una solución de cloruro de hidroxocobalamina en agua para inyección, sin excipientes adicionales, lista para administrar, sin necesidad de preparación extemporánea, trasvases o cualquier otra operación previa a la administración que comprometa la calidad y la seguridad. La estabilidad de las soluciones acuosas de hidroxocobalamina $^{21-24}$ y tamponadas ${ }^{25}$ a $\mathrm{pH} 4,3$ en ambos casos a las dosis indicadas para su actividad sobre la hematopoyesis, ha sido estudiada. Las soluciones mantuvieron al menos un 90 por ciento de hidroxocobalamina después de 170 semanas a $30^{\circ} \mathrm{C}$. Hay que añadir, en apoyo a la hipótesis del presente trabajo sobre la estabilidad de la solución de hidroxocobalamina, la experiencia adquirida en el Centro Militar de Farmacia de la Defensa de Córdoba por la producción y control de calidad del Elaborado de Farmacia Militar "Vitamina B12 Inyectable, $1 \mathrm{mg}$ en $1 \mathrm{ml}$, envase de 10 ampollas" durante más de 15 años. Su principio activo, la cianocobalamina, es el producto natural resultante de la combinación de la hidroxocobalamina con el ion cianuro. Los resultados obtenidos mediante los reanálisis efectuados en paralelo al desarrollo del presente trabajo sobre unidades conservadas en la muestroteca del CEMILFARDEF de Córdoba han confirmado la estabilidad de la solución más allá del periodo máximo de caducidad legalmente autorizado (5 años).

Este estudio sobre la estabilidad de la solución inyectable de cloruro de hidroxocobalamina en agua para inyección, como antídoto contra cianuros, permite concluir que dicha solución se ha mostrado estable durante el tiempo de estudio. El contenido en cloruro de hidroxocobalamina permanece prácticamente invariable en cualquiera de los envases estudiados cuando la temperatura de conservación es inferior a $25^{\circ} \mathrm{C}$. Aun con los resultados más desfavorables obtenidos cuando la temperatura de estudio fue $37^{\circ} \mathrm{C}$, el contenido se mantuvo dentro del rango de conformidad especificado por la farmacopea. A la temperatura de conservación estándar para la mayoría de los medicamentos $\left(25^{\circ} \mathrm{C}\right)$ no se ha detectado degradación o descomposición a productos secundarios durante el tiempo de ensayo, en algunas muestras superior a 472 días.

La baja variabilidad en los valores de la longitud de onda de máxima absorción obtenidos para cada muestra y para cada tiempo mediante los barridos ultravioleta-visible, constituye también un indicativo de la estabilidad de la solución. Han sido también de gran interés para este estudio de estabilidad los contenidos de cloruro de hidroxocobalamina obtenidos por HPLC en las muestras más envejecidas de cada uno de los cuatro lotes ensayados, así como la no aparición de picos secundarios en el desarrollo de los cromatogramas.

Las muestras sometidas a $37^{\circ} \mathrm{C}$ durante más de 15 meses sufrieron una reducción en su contenido en principio activo, aunque se mantuvo siempre dentro del rango de aceptación (95 $-115 \%)^{15}$

La solución inyectable de cloruro de hidroxocobalamina no puede ser esterilizada por calor. Los resultados obtenidos de las muestras autoclavadas a $121^{\circ} \mathrm{C}$ durante 20 minutos muestran una reducción en el contenido en principio activo y la aparición de picos secundarios en el cromatograma. La nueva solución inyectable deberá ser esterilizada mediante filtración esterilizante, 
y su proceso de fabricación realizado por vía aséptica sin esterilización terminal.

La pérdida de agua de las soluciones envasadas en bolsas de polipropileno bajo estrés de temperatura hace descartar a este envase para la solución inyectable de hidroxocobalamina. Por el contrario, los resultados fueron de conformidad tanto con los viales de COC como los de PP, gracias a sus propiedades barrera. El color rojo intenso característico de la solución inyectable de hidroxocobalamina, evidente en los envases transparentes ensayados y que produce cierta animadversión sobre el aspecto del preparado, puede ser ocultado mediante el empleo de envases de color ámbar.

Por todo lo anterior, los envases más adecuados para la solución inyectable de hidroxocobalamina, para uso en urgencias como antídoto contra cianuros, son los viales de polipropileno de 100 mililitros de color ámbar, cerrados con tapón de caucho y cápsula de aluminio tipo flip-off. Son también los más adecuados en las condiciones de empleo en el ámbito militar por sus ventajas logístico-operativas y asistenciales: envases de bajo peso, volumen y fragilidad, y facilidad de empleo. Cada vial estará etiquetado y envasado en un estuche de cartón con su prospecto correspondiente. La solución así envasada y conservada a temperatura inferior a $25^{\circ} \mathrm{C}$ podrá tener un periodo de caducidad superior a 15 meses.

A estas ventajas hay que añadir la sencillez en la tecnología farmacéutica necesaria para la producción de una solución inyectable de hidroxocobalamina, más simple que la necesaria para una presentación como polvo estéril para solución parenteral extemporánea. Así mismo, su empleo es fácil, seguro, rápido y de calidad al presentarse como solución inyectable estable y lista para administrar. Se reducen los riesgos inherentes a una preparación extemporánea (contaminación, roturas, pérdidas de solución, etc) y se gana rapidez conforme exige la situación de urgencia motivada por una intoxicación por cianuros. Se administrará directamente mediante inyección intravenosa lenta.

Desde el punto de vista estratégico, en Defensa NBQ, los resultados obtenidos permiten disponer de una alternativa al nitrito sódico como antídoto contra cianuros, especialmente cuando éste está contraindicado. En la próxima revisión del Petitorio de Farmacia del Ministerio de Defensa se propondrá, si procede, el Elaborado Hidroxocobalamina DEF Inyectable, envase de 1 vial de $100 \mathrm{ml}$ como un nuevo antídoto para Defensa NBQ, indicado en el tratamiento de urgencia de la intoxicación por cianuros en el ámbito de las Fuerzas Armadas Españolas.

\section{CONCLUSIONES}

La solución inyectable de cloruro de hidroxocobalamina en agua para inyección, a la concentración indicada como antídoto N.B.Q. contra cianuros, es estable a temperaturas inferiores a $25^{\circ}$ $\mathrm{C}$, al menos durante un periodo de 15 meses.

El contenido en cloruro de hidroxocobalamina se mantiene en el rango de aceptación, entre el 95 y el $115 \%$.
El envase más adecuado para está solución inyectable, de uso en urgencias normalmente extrahospitalarias, son los viales de polipropileno de color ámbar y $100 \mathrm{ml}$ de capacidad, por su inercia, bajo peso, volumen y fragilidad.

La solución inyectable debe ser esterilizada por filtración esterilizante y producida por vía aséptica sin esterilización terminal.

\section{BIBLIOGRAFÍA}

1. Hall AH; Dart R; Bogdan G. Sodium thiosulfate or hydroxocobalamin for the empiric treatment of cyanide poisoning?. Ann Emerg Med 2007; 49(6): 814-816.

2. Alarie Y. Toxicity of fire smoke. Crit Rev Toxicol 2002; 2: 259-289.

3. Baud FJ; Barriot, P; Riou, B. Toxicité systémique des fumes d'incendie. JEUR 1988; 1: 83-88.

4. Baud FJ, Barriot P, Toffis V, Riou B, Vicaut E, Lecarpentier Y, et al. Elevated blood cyanide concentrations in victims of smoke inhalation. New England Journal of Medicine 1991; 325: 1761-1766.

5. NATO Handbook on the medical aspects of NBC Defensive Operations AMedP-6(C) Vol. 3 (Chemical). 2005.

6. Sauer SW; Keim ME. Hydroxocobalamin: improved public health readiness for cyanide disasters. Ann Emerg Med 2001; 37(6): 635-41.

7. Gracia R; Shepherd G. Cyanide poisoning and its treatment. Pharmacotherapy 2004; 24(10): 1358-1365.

8. Rotenberg JS. Cyanide as a weapon of terror. Pediatr Ann 2003; 32:236-240.

9. Keim ME. Terrorism involving cyanide: the prospect of improving preparedness in the prehospital setting. Prehospital Disaster Med 2006; 21:s56-s60.

10. http://emedicine.medscape.com/article/814287-overview Fecha último acceso web: 02/01/2012.

11. Mégarbane B; Delahaye A; Goldgran-Tolédano D; Baud FJ. Antidotal treatment of cyanide poisoning. J Chin Med Assoc 2003; 66(4): 193-203.

12. Petitorio de Farmacia del Ministerio de Defensa. Orden Ministerial número 53/2004, de 18 de Marzo (B.O.D número 63, de 31 de Marzo de 2004)

13. Baskin SI; Horowitz AM; Nealley EW. The antidotal action of sodium nitrite and sodium thiosulfate against cyanide poisoning. J Clin Pharmacol 1992; 32(4): 368-375.

14. http://www.cyanokit.com Fecha último acceso web: 02/01/2012.

15. The United States Pharmacopeia XXI Edition. 512-514.

16. European Pharmacopoeia 7.0 Edition. 2010; 2204-2205.

17. Farmacopea Española $2^{\text {a }}$ Edición. 2002: 293-295.

18. WHO Technical Report Series, No. 953, 2009, Annex 2: Stability testing of active pharmaceutical ingredients and finished pharmaceutical products. http:// www.who.int/medicines/publications/pharmprep/pdf_trs953.pdf\#page=101 Fecha último acceso web: 02/01/2012.

19. Kirk MA; Gerace R; Kulig KW. Cyanide and methemoglobin kinetics in smoke inhalation victims treated with the cyanide antidote kit. Ann Emerg Med 1994; 24(3): 539-40.

20. https://sinaem4.agemed.es/consaem/fichasTecnicas.do?metodo=buscar Centro de Información online de Medicamentos de la AEMPS - CIMA (hidroxocobalamina). Fecha último acceso web: 02/01/2012

21. Ceccarini R; Cingolani E; Princivalle M. Stability of aqueous solutions of hidroxocobalamin. Il Farmaco; edizione pratica 1963; 18: 600-618.

22. Casadio S; Sekules G; Cappa E. Stability of aqueous solutions of hydroxocobalamin: III. Il Farmaco; edizione pratica 1962; 17: 611-619.

23. Casadio S; Sekules G; Cappa E. Stability of aqueous solutions of hydroxocobalamin: II. Il Farmaco; edizione pratica 1962; 17: 603-610.

24. Casadio S; Sekules G; Cappa E. Stability of aqueous solutions of hydroxocobalamin: I. Il Farmaco; edizione pratica 1962; 17: 593-602.

25. Marcus AD; Stanley JL. Stability of the cobalamin moiety in buffered aqueous solutions of hydroxocobalamin. J Pharm Sci 1964; 53(1): 91-94. 